\title{
An Efficient Data-driven Tissue Deformation Model
}

\section{Mosbech, Thomas Hammershaimb; Ersbøll, Bjarne Kjær; Christensen, Lars Bager}

Published in:

2009 IEEE 12th International Conference on Computer Vision Workshops (ICCV Workshops)

Link to article, DOI:

10.1109/ICCVW.2009.5457497

Publication date:

2009

Document Version

Publisher's PDF, also known as Version of record

Link back to DTU Orbit

Citation (APA):

Mosbech, T. H., Ersbøll, B. K., \& Christensen, L. B. (2009). An Efficient Data-driven Tissue Deformation Model. In 2009 IEEE 12th International Conference on Computer Vision Workshops (ICCV Workshops) (pp. 17711777). IEEE. https://doi.org/10.1109/ICCVW.2009.5457497

\section{General rights}

Copyright and moral rights for the publications made accessible in the public portal are retained by the authors and/or other copyright owners and it is a condition of accessing publications that users recognise and abide by the legal requirements associated with these rights.

- Users may download and print one copy of any publication from the public portal for the purpose of private study or research.

- You may not further distribute the material or use it for any profit-making activity or commercial gain

- You may freely distribute the URL identifying the publication in the public portal 


\section{An Efficient Data-driven Tissue Deformation Model}

\author{
Thomas Hammershaimb Mosbech, Bjarne Kjær Ersbøll \\ Technical University of Denmark \\ Kgs. Lyngby, DK-2800 \\ $\{\mathrm{tm}, \mathrm{be}\} @ i m m . d t u . d k$
}

\author{
Lars Bager Christensen \\ Danish Meat Association \\ Roskilde, DK-4000 \\ 1bc@danishmeat.dk
}

\begin{abstract}
In this paper we present an efficient data-driven tissue deformation model. The work originates in process automation within the pig meat processing industry. In the development of tools for automating accurate cuts, knowledge on tissue deformation is of great value. The model is built from empirical data; 10 pig carcasses are subjected to deformation from a controlled source imitating the cutting tool. The tissue deformation is quantified by means of steel markers inserted into the carcass as a three-dimensional lattice. For each subject marker displacements are monitored through two consecutive computed tomography images - before and after deformation; tracing corresponding markers provides accurate information on the tissue deformation. To enable modelling of the observed deformations, the displacements are parameterised applying methods from point-based registration. The parameterisation is based on compactly supported radial basis functions, expressing the displacements by parameter sets comparable between subjects. For modelling the tissue deformation, principal component analysis is applied, treating each of the parameter sets as an observation. Using leave-one-out cross-validation, marker displacements are estimated in all subjects from the mean parameters. This yields an absolute error with mean $1.41 \mathrm{~mm}$. The observed lateral movement of the loin muscle is analysed in relation to the principal modes, and the results are compared to manual measurements of carcass composition. We find an association between the first principal mode and the lateral movement. Furthermore, there is a link between this and the ratio of meat-fat quantity - a potentially very useful finding since existing tools for carcass grading and sorting measure equivalent quantities.
\end{abstract}

\section{Introduction}

In the meat industry there is increasing interest in developing tools for process automation. The traditional manual slaughtering procedures give rise to a labour-intensive working environment with physically demanding tasks and repetitive work performed under pressure. The goals of efficient automated processing include safer and faster production, better hygiene, more uniform quality of the end products, and in the end, better returns for abattoirs.

Existing automation tools in pig meat production include systems for classifying and sorting carcasses, as well as robotics for evisceration, carcass cutting, boning and trimming various parts. Technologies currently used here are mostly computer vision and ultrasonics [7, 9]. An upcoming technology in automation is the use of computed tomography $(\mathrm{CT})$ to achieve detailed information on the raw materials. Both in measuring general tendencies of populations, but also for on-line use in the abattoirs.

One hurdle in the development of the robotics is to handle the large biological variation present in the raw material - automatically adapting the processing to each individual subject. Within this area, an important topic is prediction of subject behaviour during handling, e.g. fixing a piece of meat for cutting can induce tissue deformations making it difficult for the machine to perform accurate cuts.

In this paper we use CT images as guidance in the development of a tool for processing pig backs to produce bacon with a given fat-layer thickness; homogeneous products from inhomogeneous raw materials. Figure 1 shows a 3-dimensional model of a pig back created from a CT image. Figure 1(a) shows the model with a distinction between the meat and the rind (facing down). Figure 1(b) shows the model after a 'virtual cut' has been performed, leaving only the rind. From the model we can see that the transition between fat and meat is far from smooth, thus a robot trimming the fat layer must be able to adjust to the shape of each individual subject [1].

Initial stages of the development process have identified the need for information on the tissue deformations caused by handling the pig back. Pressure from the knives trimming the rind tends to cause lateral movement in the loin muscle, making it difficult to perform accurate cuts. We study a simplified version of this by applying pressure from a single bolt on the skin surface and examining the induced tissue deformation in the area around the loin. 


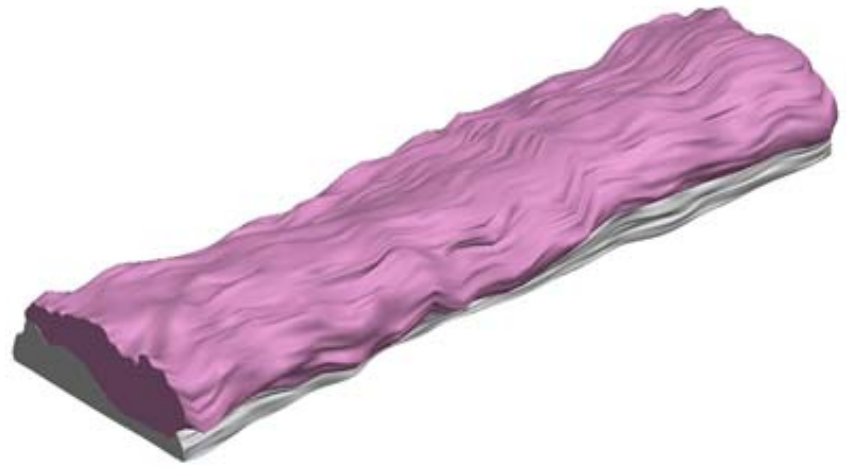

(a)

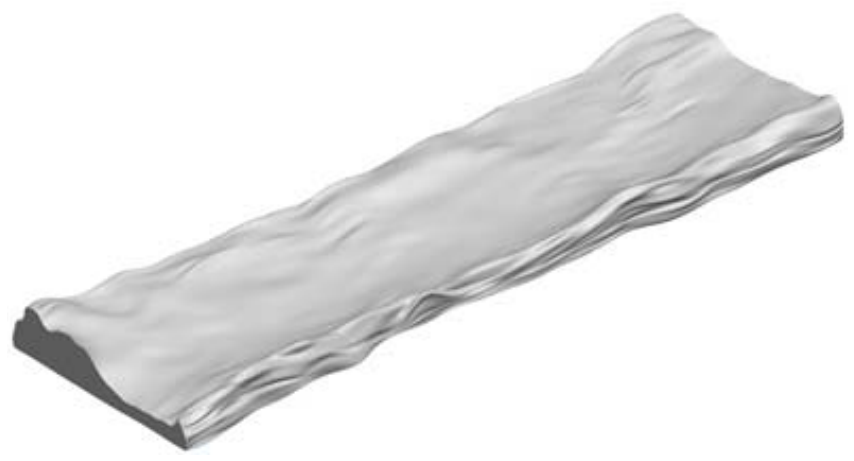

(b)

Figure 1. 3D model of a pig back created from a CT scan. (a) Model distinguishing between the loin and streaky (top) and the rind (bottom). (b) 'Virtual cut' showing only the rind layer; the variation of the contours in the transition between meat and rind is evident.

Tissue deformation modelling is a widespread area of research within medical imaging, e.g. haptic interfaces with realistic feedback used in simulation tools for surgical training.

A widely used approach for implementing these models is the use of finite element methods with material properties determined from both in vivo and ex vivo experiments. A thorough overview of such continuum-mechanical models is presented by Famaey and Sloten in [4] in relation to applications in virtual surgery and surgical robotics. Another approach is the use of basis functions to model tissue deformations - a method related to image registration, forming the warp function as sums of smooth basis functions. Wachowiak et al. [10] describe an application to needle insertion simulation in prostate brachytherapy using radial basis functions with compact support.

Recently, Mosbech et al. [8] presented a method for accurate quantification of soft tissue deformation, they proposed a technique based on CT images of soft tissue with inserted steel markers. We use this method in our datadriven analysis of tissue deformation. Markers are placed inside the tissue and the tissue is subjected to deformation. The resulting marker movements are monitored using CT imaging, enabling automatic generation of accurate landmarks. The observed landmark displacements are then used to analyse the induced soft tissue deformation.

\section{Data Acquisition}

For the experiments we used 10 pig backs of varying size and composition (quantity of fat and meat). In each subject a number of steel markers were inserted with a syringe through the skin. The steel markers had a diameter of 0.5 $\mathrm{mm}$. The insertions were performed such that the markers resembled a 3-dimensional lattice of approximately 180 $\mathrm{mm} \times 40 \mathrm{~mm} \times 70 \mathrm{~mm}$. The number of inserted mark- ers varied between 113 and 140 because of different subject sizes.

We mounted the bolt for inducing the tissue deformation on a nylon plate. Each pig back subject was put on the plate with the rind-side facing down. The subject was aligned, such that the bolt was positioned on the skin in the middle of the lattice of injected markers. On the meat-side of the subject we placed a soft cushion to prevent the subject from being dislodged unintentionally. For each subject we did two consecutive CT scans; one without any deformation induced by the bolt, and one pressing the bolt $20 \mathrm{~mm}$ against the skin surface.

The images were acquired with approximately isotropic voxels; $0.9375 \mathrm{~mm} \times 0.9375 \mathrm{~mm}$, with a slice thickness of $1 \mathrm{~mm}$. Figure 2 shows examples of the image data.

\section{Methods}

Compared to the surrounding soft tissue, the steel markers lead to very high intensities in the CT images. The positions can therefore be extracted accurately by intensity thresholding.

For each subject this gives two point sets of size $n$ in $d=3$ dimensions; $\mathcal{P}$ and $\mathcal{Q} \in \mathbb{R}^{n \times d}$, holding marker positions retrieved from the two images with and without deformation, respectively.

To automatically achieve point-correspondences within each subject, we apply the Hungarian Algorithm [6], matching point pairs in the two sets by minimising the sum of the Euclidean distances.

As the 10 resulting sets of pairs differ in both size and marker positions, no point-correspondences can be established between subjects. Thus the tissue deformations contained in the marker displacements are difficult to compare across subjects. To facilitate comparison we parameterise the marker displacements, bringing them into a common 


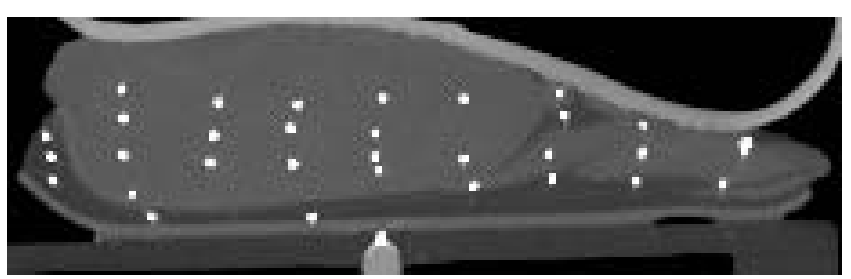

(a)

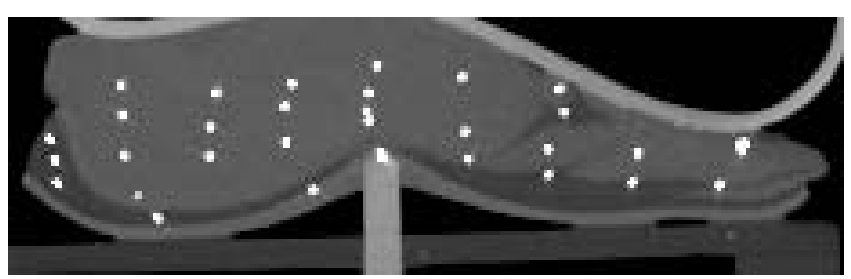

(b)

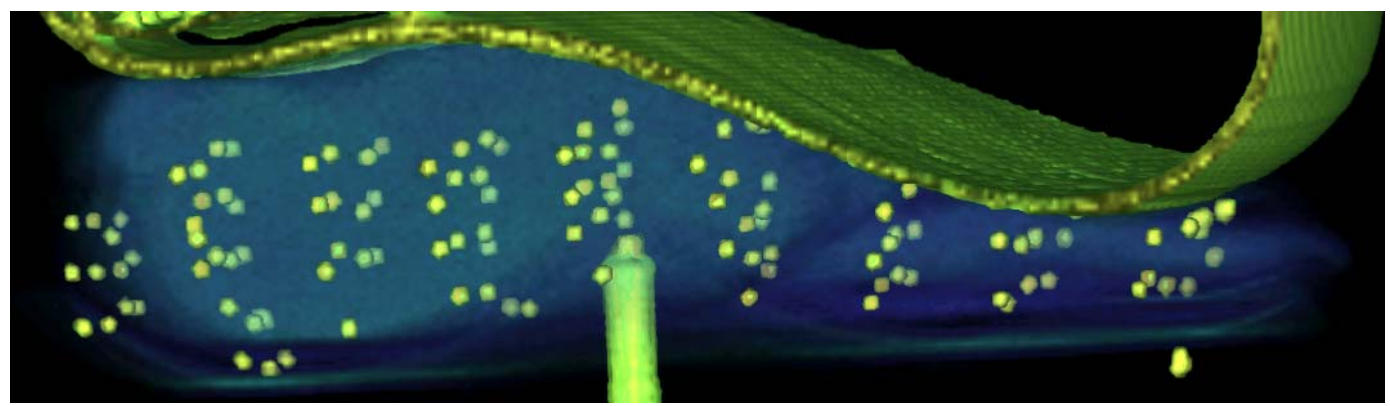

(c)

Figure 2. (a) and (b) MIPs of the slices around the bolt showing the subject without and with deformation. The high intensity of the markers makes them distinguishable from the low-intensity soft tissue. The high intensity band at the top is the air-filled soft cushion. (c) Three-dimensional visualisation of the deformed image, the view is slightly rotated and the opacity of the soft tissue has been decreased to illustrate the marker grid structure.

reference frame.

The parameterisation can be regarded as a task from point-based registration; determining a geometrical transformation aligning point-pairs by estimating displacements. We formulate this alignment of two corresponding points $\mathbf{p}_{i} \in \mathcal{P}$ and $\mathbf{q}_{i} \in \mathcal{Q}$ as a function $\phi$ of parameters $\mathbf{x} \in \mathbb{R}^{d \cdot n_{k}}$

$$
\phi\left(\mathbf{p}_{i}, \mathbf{x}\right)=\mathbf{p}_{i}+\mathbf{u}\left(\mathbf{p}_{i}, \mathbf{x}\right)
$$

modelling the displacements by the parametric transformation $\mathbf{u}:\left(\mathbb{R}^{d}, \mathbb{R}^{d \cdot n_{k}}\right) \rightarrow \mathbb{R}^{d}$.

The transformation is composed of a sum of basis functions $\psi$ defined on a set of knot points $\mathcal{C} \in \mathbb{R}^{n_{k} \times d}$ all within the spatial range of $\mathcal{P}$. For a single point the displacement in one direction is the sum of $n_{k}$ basis functions

$$
u\left(\mathbf{p}_{i}, \mathbf{x}\right)=\sum_{j=1}^{n_{k}} x_{j} \psi\left(r_{j}\right),
$$

where $r_{j}=\left\|\mathbf{p}_{i}-\mathbf{c}_{j}\right\|$; the Euclidean distance between the point $\mathbf{p}_{i} \in \mathcal{P}$ and knot $\mathbf{c}_{j} \in \mathcal{C}$.

As basis functions we use a certain type of radial basis function with compact support $\psi_{d, k} \in C^{2 k}(\mathbb{R})$ as introduced by Wendland [11]. In [5] Fornefett et al. describe an application of these functions to model deformations in relation to registration of medical images. For dimension $d=3$ and smoothness $k=2$ the Wendland-basis function is formulated as

$$
\psi_{3,2}=(1-r)_{+}^{6}\left(35 r^{2}+18 r+3\right) .
$$

In this univariate polynomial, the compact support is controlled by $(1-r)_{+}^{l}=(1-r)^{l}$ for $0 \leq r \leq 1$ and 0 for $r>1$. A parameter $a>0$ can be applied for scaling this locality; $\psi(r) \equiv \psi(r / a)$. That is, the global effect of $\psi$ increases with $a$, as all points within distance $a$ from a particular knot are affected.

The optimal vector of parameters $\mathbf{x} \in \mathbb{R}^{n_{k}}$ to represent one direction of the tissue deformation contained in all pairs in $\mathcal{P}$ and $\mathcal{Q}$ for one subject is determined by minimising a sum of $n$ squared pair-wise Euclidean distances between observations and estimates

$$
\underset{\mathbf{x}}{\operatorname{argmin}} \sum_{i=1}^{n}\left\|\mathbf{q}_{i}-\phi\left(\mathbf{p}_{i}, \mathbf{x}\right)\right\|^{2} .
$$

With (1) and (2) we can construct the estimates as a linear system of equations

$$
\hat{\mathbf{q}}=\mathbf{p}+\mathbf{A x},
$$

where $\mathbf{p}, \hat{\mathbf{q}} \in \mathbb{R}^{n}$ and $\mathbf{A}$ is an $n \times n_{k}$ matrix of basis function values $\{A\}_{i j}=\psi_{d, k}\left(\left\|\mathbf{p}_{i}-\mathbf{c}_{j}\right\|\right)$. The system can be expanded to estimate displacements in all three dimensions;

$$
\hat{\mathbf{q}}=\mathbf{p}+\left[\begin{array}{ccc}
\mathbf{A} & \mathbf{0} & \mathbf{0} \\
\mathbf{0} & \mathbf{A} & \mathbf{0} \\
\mathbf{0} & \mathbf{0} & \mathbf{A}
\end{array}\right] \mathbf{x},
$$

with $\mathbf{p}, \hat{\mathbf{q}} \in \mathbb{R}^{d \cdot n}$ and parameters $\mathbf{x} \in \mathbb{R}^{d \cdot n_{k}}$. This can be solved using standard optimisation techniques, e.g. by 


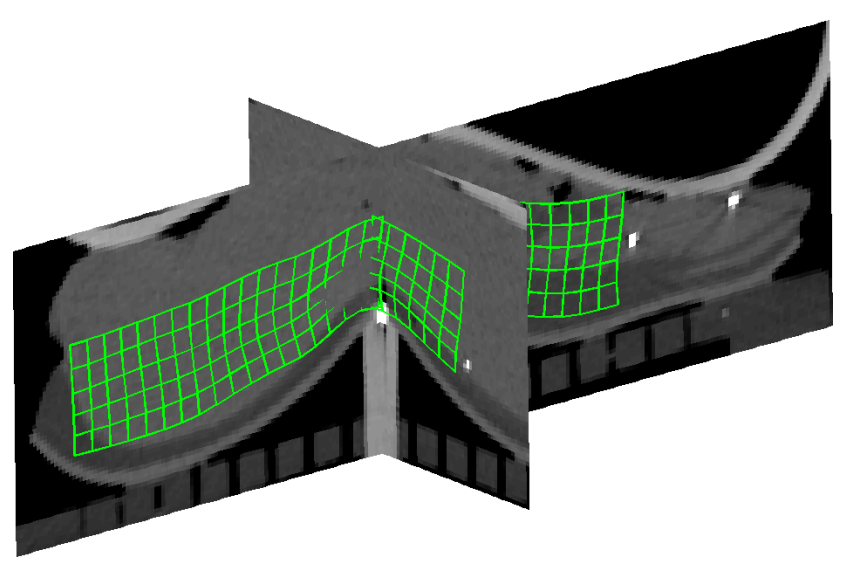

Figure 3. A mesh of points with $5 \mathrm{~mm}$ spacing deformed under the estimated parameters. The mesh is superimposed on two planes of the CT image. As the deformation is three-dimensional, part of the grid is disappears into the image slice from this perspective.

minimising the cost function equivalent to (4) w.r.t. $\mathbf{x}$

$$
f=\frac{1}{2}\left(\mathbf{q}-\left(\mathbf{p}+\mathbf{A}_{0} \mathbf{x}\right)\right)^{T}\left(\mathbf{q}-\left(\mathbf{p}+\mathbf{A}_{0} \mathbf{x}\right)\right)
$$

where $\mathbf{A}_{0}$ is the block diagonal matrix of (6).

This parametric representation of the observed marker displacements enables estimation of the tissue deformation between the markers. Applying the same basis function parameters and set of knot points, the $\mathbf{A}$ matrix of (5) is computed with respect to these new points of interest (e.g. all voxel positions). The parameters can then be plugged into (6) for computing the displacements. Figure 3 shows an example.

\section{Analysis}

As mentioned earlier, the $N=10$ tissue deformations are comparable by means of their parameter vectors, when the same parameterisation has been applied on all subjects. This implies using the same values of $a, d$ and $k$, but also a common knot set-up $\mathcal{C}$. To handle this knot-setup, a steel marker placed on the tip of the pressure bolt is used as origin for consistent positioning of the knot-grid on all subjects.

We analyse the variability of the estimated parameters by applying principal component analysis (PCA), building a general model with methods from statistical shape modelling [3].

Regarding each of the estimated parameter vectors as an observation of a random variable, we estimate the mean

$$
\overline{\mathbf{x}}=\frac{1}{N} \sum_{s=1}^{N} \mathbf{x}_{s}
$$

and calculate the covariance matrix of the parameters with the mean subtracted

$$
\boldsymbol{\Sigma}=\frac{1}{N-1} \sum_{s=1}^{N}\left(\mathbf{x}_{s}-\overline{\mathbf{x}}\right)\left(\mathbf{x}_{s}-\overline{\mathbf{x}}\right)^{T}
$$

The eigenvectors and eigenvalues of $\boldsymbol{\Sigma}$ are then estimated, and the eigenvalues are sorted non-increasing; $\lambda_{1} \geq \lambda_{2} \geq$ $\cdots \geq \lambda_{d \cdot n_{k}}$. The corresponding eigenvectors are gathered in a matrix forming an orthonormal basis; $\mathbf{P}=$ $\left[\mathbf{P}_{1} \mathbf{P}_{2} \cdots \mathbf{P}_{d \cdot n_{k}}\right]$. These vectors are the principal modes forming the model for approximating a parameter vector; adding the mean and a linear combination of modes

$$
\mathbf{x}=\overline{\mathbf{x}}+\mathbf{P}^{\prime} \mathbf{b}^{\prime}
$$

where $\mathbf{P}^{\prime}$ is a subset of $\mathbf{P}$ containing the $l$ first modes, and $\mathbf{b}^{\prime} \in \mathbb{R}^{l}$ is the model parameters.

Using leave-one-out cross-validation, $N$ models are estimated - each from $N-1$ observations. We assess the generality of the model by considering the simplest model consisting of the mean deformation parameters only. Instead of applying the transformations on regular grids as shown in figure 3, we apply them to the set of marker positions corresponding to the parameter set left out in building the model. In this way we can estimate the prediction error of the model, comparing estimated and observed displacements. This yields an absolute error of $1.41 \pm 0.87 \mathrm{~mm}-$ comparable to the voxel size of the images.

To examine the effects of the modes in detail, the zeromean parameter vector is projected onto the full set of modes

$$
\mathbf{b}=\mathbf{P}^{T}(\mathbf{x}-\overline{\mathbf{x}}),
$$

the deformation parameters are then reconstructed using only the model parameters and modes of interest as in (10).

We take an approach slightly different from the model formulated in (10) and (11). No cross-validation is applied, and the mean is disregarded, as its effect on the deformations is common for all observations. In the PCA model the amount of the data set's variation represented by the $i$ th mode is proportional to the relative size of the $i$ th eigenvalue. The graph in figure 4 relates the number of modes in the model to the percentage of variance explained. We show only the first nine modes - explaining the full variance - as the model is built from 10 observations.

The three first modes cover approximately two-thirds of the total variance. With the approach described above, we examine the effect on the tissue deformation of each of the modes individually. Figure 5 shows an example of the results; using the reconstructed deformation parameters, we calculate displacements for a grid of points - covering the range of the knots and markers - and visualise them as arrows superimposed on the corresponding image slice.

For the first three modes, the estimated displacements show no general tendencies in the streaky-part of the backs 


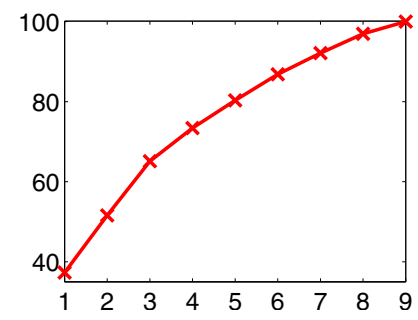

Figure 4. Graph showing the percentage of variance explained by the principal modes. The nine first modes are used to explain all of the variance, as the model is built from 10 observations.

(rightmost on the example in figure 5). As this part exhibits a highly variable anatomical structure throughout the 10 subjects, it is difficult for the model to generalise these deformations.

Regarding the estimated point displacements in the loin muscle, the first mode - explaining $37 \%$ of the total variance - shows a relation to the degree of lateral movement for all subjects. The movement appears to be most profound in the tissue around the tip of the pressure bolt, but on some subjects it propagates even further into the muscle. As mentioned in the introduction, this lateral movement is of particular interest in the development of automated tools for precise rind trimming. The movement complicates the process of producing a product with a given fat layer thickness.

We focus the analysis on this first principal mode and examine the resulting displacements of points within the loin muscle and in the rind layer beneath it. The points are selected by means of manual segmentations of the loin muscle.

Existing carcass grading systems are capable of performing measurements of the fat and loin muscle thickness [2], so it is sensible to use these two measurements to characterise the carcass composition of the subjects - that is, for each subject we also perform a manual segmentation to assess the mean fat layer thickness. Figure 6 relates the estimated lateral movements to the ratio between loin size and mean fat layer thickness. For subjects 2 and 9 - with notable positive displacement means - the loin muscle is not moving to the right, since the left-directed deformation contained in the mean is disregarded in the analysis. From the figure we see, that the lateral movement differs clearly from the rest on the most lean and fat subjects. This indicates the possibility of sorting the pig backs by means of the loin-fat ratio and discriminating the processing $-e . g$. by applying uneven pressure on the meat side to compensate for the lateral movement of the muscle.

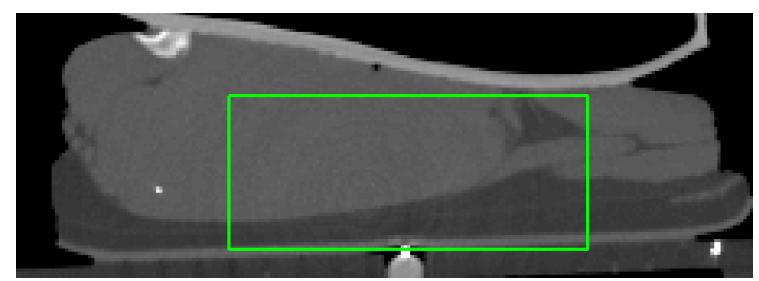

(a)
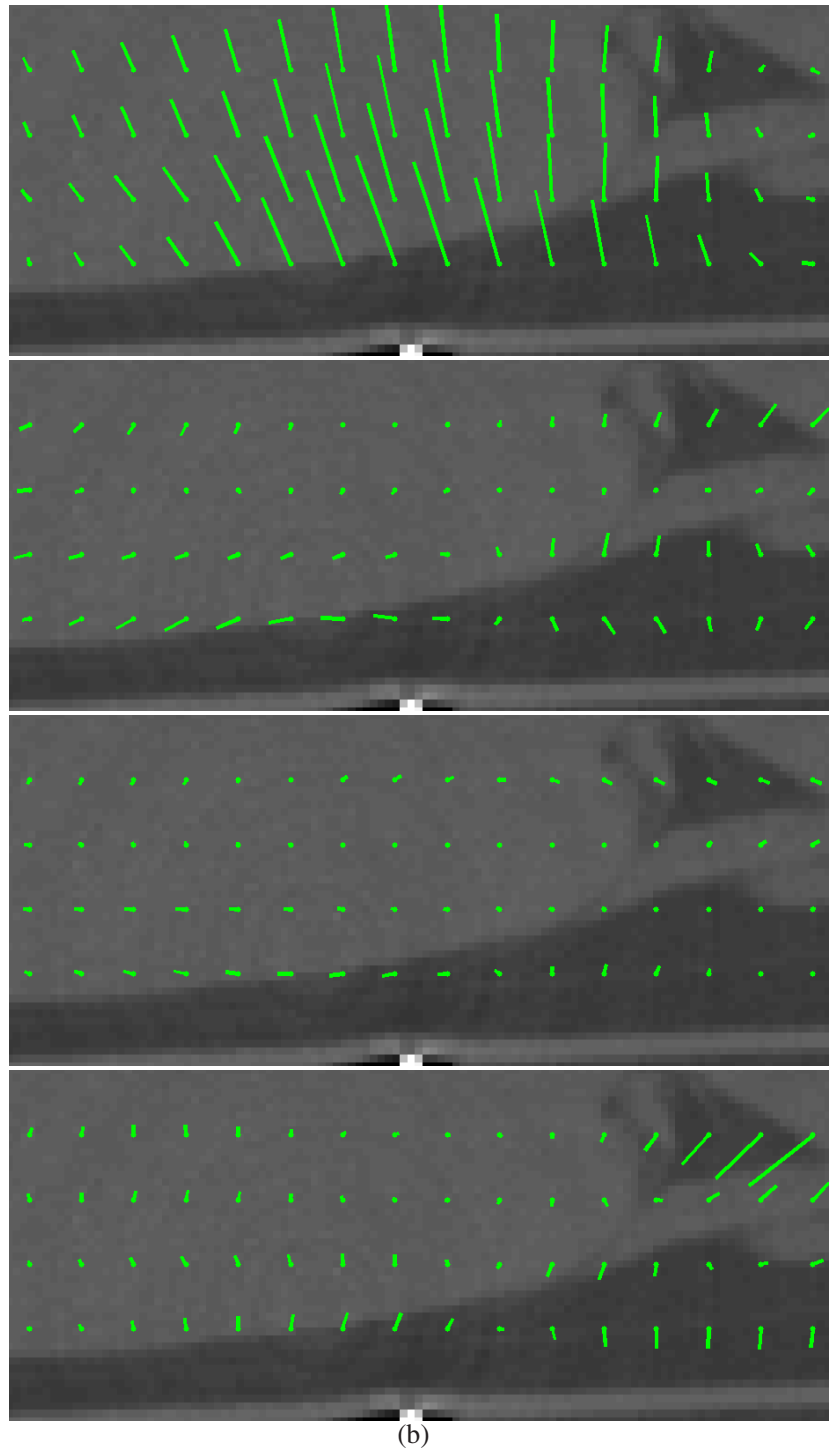

Figure 5. The effect of the mean and each of the three first principal modes. (a) The displacements are illustrated on a single slice zoomed to a region around the pressure bolt containing parts of the streaky (right) and loin muscle (left). (b) Four slices; the displacements are estimated for a grid of points and shown as lines superimposed on the image (from top to bottom; mean, modes 1 , 2 and 3). For illustrative purposes the lines corresponding to the three single modes have been magnified by a factor of five. 


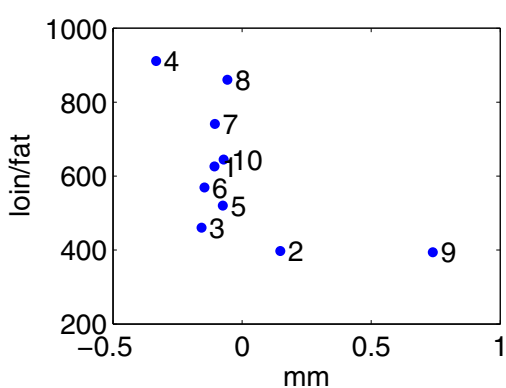

(a)

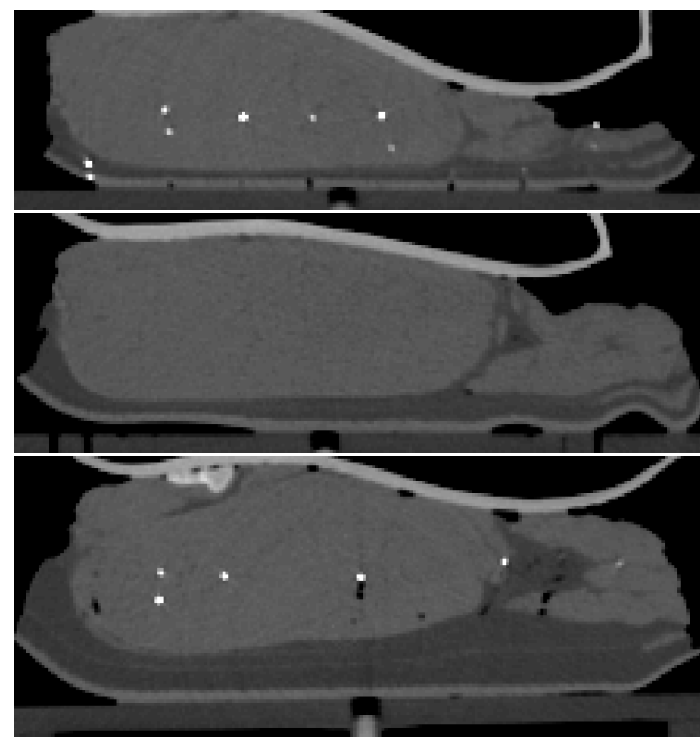

(b)

Figure 6. Analysing the first principal mode regarding displacements estimated for points within the loin muscle and in the fat layer beneath it. (a) Plot relating lateral movement ( $x$-axis) and ratio between the manual measurements of loin size and mean fat layer thickness ( $y$-axis). (b) Illustrating three different carcass compositions resulting in different lateral loin muscle movements. From top to bottom the slices are subjects 4,1 and 9 .

\section{Conclusion}

This paper described an efficient method for data-driven modelling deformation of soft tissue. The model is based on three-dimensional computed tomography images of 10 pig carcasses subjected to deformation. The tissue deformation is accurately quantified by steel markers inserted into the carcass. The observed displacements from each subject are transformed into a common reference frame with a parametric representation of radial basis functions with compact support.

The derived parameters are compared using principal component analysis, forming a tissue deformation model. The generalisation of the model is assessed by estimating marker displacements from the mean parameters using leave-one-out cross-validation by subject. Across all subjects the model achieves an absolute error of $1.41 \pm 0.87$ $\mathrm{mm}$. The first principal mode of the model covers $37 \%$ of the total variance in the data. This mode is associated with the lateral movement of the loin muscle. The magnitude of this movement is compared with measurements of carcass composition; the ratio between loin size and mean fat layer thickness. The comparison shows a link; lean carcasses exhibit a higher degree of lateral loin muscle movement compared to more fatty carcasses.

In pig meat production, the presented method can provide valuable knowledge in relation to developing tools for automated processing. Our analyses are of particular interest in relation to trimming the rind of pig backs producing a product with a given fat layer thickness.

\section{References}

[1] P. Black. Millimeterpræcis afsværing af svinekamme med robot. Danish Meat Association, Ny viden om slagteri og forcedling, 2, April 2009.

[2] Carometec Food Technology. AutoFom - Fully Automatic Ultrasonic Carcass Grading for Pork Slaughterhouses. www.carometec.com.

[3] T. F. Cootes, D. H. Cooper, C. J. Taylor, and J. Graham. Active shape models - their training and application. Computer Vision and Image Understanding, 61(1):38-59, December 1995.

[4] N. Famaey and J. V. Sloten. Soft tissue modelling for applications in virtual surgery and surgical robotics. Computer Methods in Biomechanics and Biomedical Engineering, 11(4):351-366, August 2008.

[5] M. Fornefett, K. Rohr, and H. S. Stiehl. Radial basis functions with compact support for elastic registration of medical images. Image and Vision Computing, 19:87-96, 2001.

[6] H. W. Kuhn. The Hungarian method for the assignment problem. Naval Research Logistics Quarterly, 2:83-97, 1955.

[7] K. B. Madsen and J. U. Nielsen. Meat processing: Improving quality, chapter 14. Woodhead, 2002.

[8] T. H. Mosbech, B. K. Ersbøll, and L. B. Christensen. Quantification and validation of soft tissue deformation. In Society of Photo-Optical Instrumentation Engineers (SPIE) Conference Series, volume 7262 of Society of Photo-Optical Instrumentation Engineers (SPIE) Conference Series, Feb. 2009. 
[9] E. V. Olsen. Den nye AutoFOM DK sikrer mere præcis kødprocentmåling og sortering. Danish Meat Association, Ny viden om slagteri og forcedling, 2, April 2009.

[10] M. P. Wachowiak, X. Wang, A. Fenster, and T. M. Peters. Compact support radial basis functions for soft tissue deformation. In ISBI, pages 1259-1262. IEEE, 2004.

[11] H. Wendland. Piecewise polynomial, positive definite and compactly supported radial functions of minimal degree. Advances in Computational Mathematics, 4(1):389-396, December 1995. 